\title{
SPATIAL MOTION OF THE AIRCRAFT MANOEUVRING TO AVOID MOVING OBSTACLE
}

\author{
JeRZy GRAFFstein \\ Institute of Aviation, Centre of Space Technologies Department, Warsaw, Poland \\ e-mail: jgraff@ilot.edu.pl
}

\begin{abstract}
In the paper, mathematical relationships which are used to describe kinematic variables of the aircraft-obstacles configuration and motion of the aircraft are presented. These define the base for the set of conditions enabling determination of the possibility and threat of collision. The second important aim of such a definition is creation of prerequisites for selection of an appropriate anti-collision manoeuvre, computation of reference signals and inequalities used as limitations on these signals in the automatic flight control process. Theoretical analysis is illustrated by an example of computer simulation of the flight of aircraft. Two anti-collision manoeuvres are studied in this experiment. The first one, performed in a vertical plane, consists in emergency climbing. The second one, performed in the horizontal plane, is shaped by three turns, each one of small radius, to go around the obstacle and then return to the previously realised flight path.
\end{abstract}

Keywords: anti-collision manoeuvre, obstacle avoidance, flight simulation

\section{Introduction}

Recently, one can notice the growing number of methods presented in accessible scientific materials aimed at solving the collision avoidance problem with several types of obstacles. This results from growing requirements for flight safety of piloted and unmanned flying objects. The proposed solutions and mathematical methods focused on the problem differ one from another due to geometrical representations of obstacles (Graffstein, 2012a; Park et al., 2012), types of moving objects, types of obstacles, methods of getting information about obstacles (Ariyur et al., 2005; Higuchi et al., 2012), etc.

A safe preselected anti-collision manoeuvre is the most typical solution of the considered problem. Safety of such motion consists, among others, in keeping the minimum distance between any point of the object and any point of the obstacle above the assumed level, defined as the safety margin rCMB. This value depends on a number of factors, some of them were described bz Blajer and Graffstein (2012), Graffstein (2006, 2012b). The capability of avoiding the collision with previously unknown obstacles safely depends on many factors including, first of all manoeuvring capabilities of the flying object, configuration and dimension of obstacles, parameters of its motion, object-to-obstacle distance at the moment of obstacle detection and accuracy of accessible data characterising the obstacle.

The object-to-obstacle distance at the moment of obstacle detection is determined by technical means used in the obstacle detector, first of all by the type of sensor (Fasano et al., 2010; Freeman and Moosbruggerb, 2010; Higuchi et al., 2012). The knowledge of this distance and parameters of the object and obstacle motion enables determination of the most convenient anti-collision manoeuvre and its parameters (Becker et al., 2006; Graffstein, 2012a; Schøler et al., 2009). In the case of danger of collision with a moving obstacle, the situation appears to be more complex, because of possible and crucial variety of the obstacle motion. Four possible 
scenarios of such a situation exist: continuation of the flight without any changes to motion parameters and the following three types of manoeuvres: a random one or according with rules and agreed with the pilot. This last scenario can be a solution in the case of collision threat with many moving obstacles (Carbone et al., 2006; Lalish et al., 2009; Seo et al., 2012). Another scenario is assumed for a obstacle continuing its motion without any changes and reactions to the collision threat. In such a case, computation of necessary kinematic variables, described in further parts of the work, is obligatory as well as verification of collision threat conditions and choice of the appropriate manoeuvre. These operations are within the scope of tasks defined for anti-collision systems, which is described in the next Section. Presented results of computer flight simulation illustrate a practical solution of the described problem. In the numerical experiments, two different types of anti-collision manoeuvres have been simulated, both effective in avoiding collision with moving obstacle.

\section{Structure of the anti-collision system}

Preparation and execution of a manoeuvre to avoid a moving obstacle is a complex operation that constitutes considerable workload for the aircraft pilot. Thus, the structure of an anti-collision system is proposed, aiming at reduction of the workload of the pilot performing such tasks. The diagram (Fig. 1) presents the general idea of sub-system cooperation. The system is autonomous and makes use of two sources of data of obstacles: the detector of obstacles and the data base containing data of obstacles. The first source detects moving and fixed obstacles, delivers estimates of the object-to-obstacle distance and relative velocity. The data base contains information about fixed obstacles in the terrain covering the planned flight path and also about height of the terrain. This source of data plays a supplementary role in the system designed to avoid moving obstacles. During the process of searching for the appropriate anti-collision manoeuvre, the data base makes the system capable of eliminating manoeuvre candidates which make the risk of collision with one of the existing fixed obstacles and being too high.

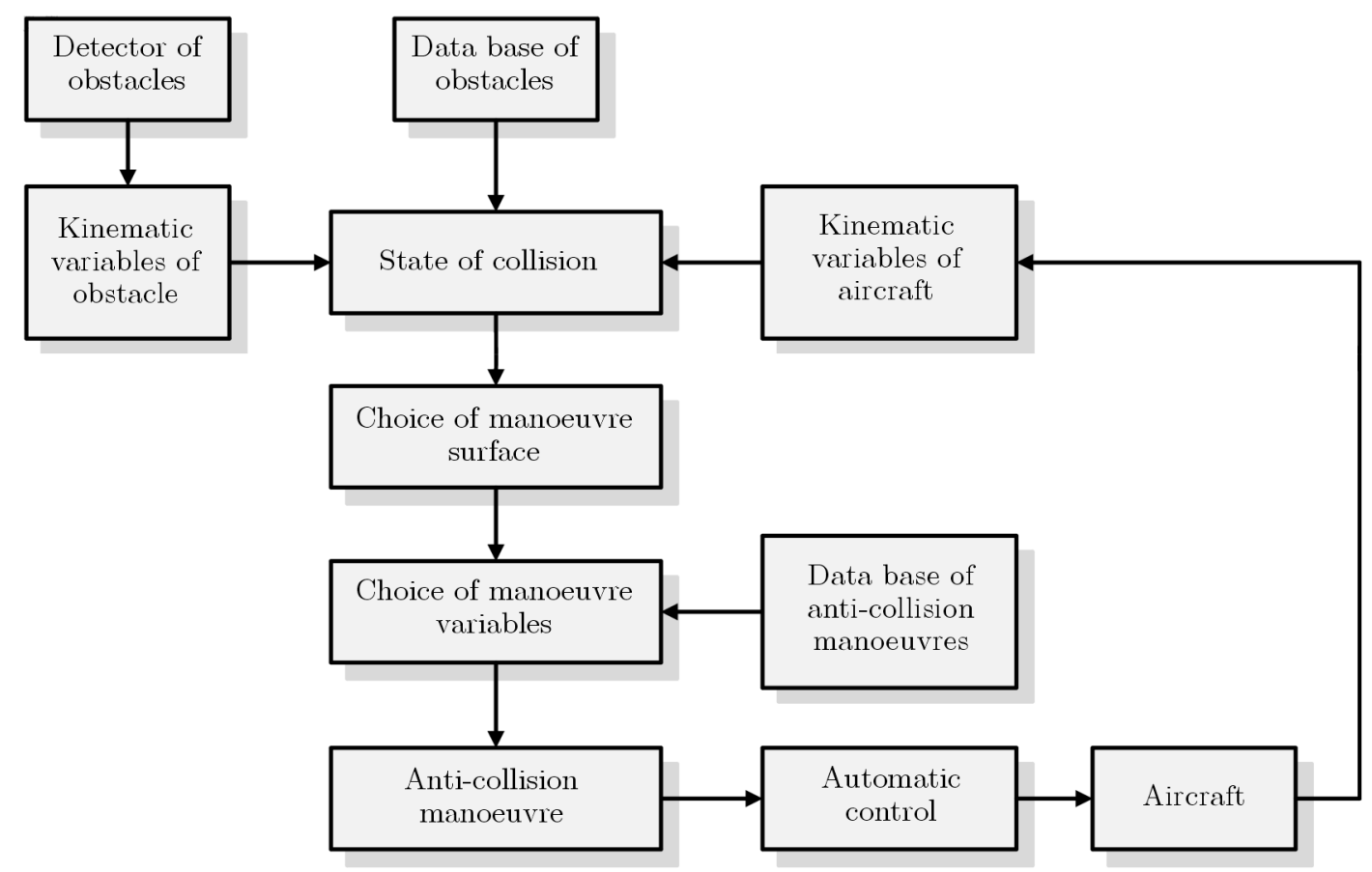

Fig. 1. Schematic diagram illustrating operation of the anti-collision system 
In every time step, when new data of the obstacles are acquired, the process of new obstacles detection is executed as well as the estimation of risk of collision in one or both planes. If the threat is detected, the procedure of searching for possible and safe anti-collision manoeuvre is executed. Searching for parameters fulfilling the appropriate criteria is performed within the base of anti-collision manoeuvres prepared in advance. In the case when more than one manoeuvre is found (for example: performed within both planes), selection of one of them is necessary. Safety is the basic criterion for the final decision, so is the magnitude of safety margin, among others. On the other side, important conditions require minimisation of two values: the distance from the previously planned flight path and the time interval defined as becoming when the aircraft abandons this flight path and ends when returns. In addition, flight conditions have to be taken into account: the magnitude and type of disturbances, e.g. wind direction and speed, altitude, height of the obstacle, etc. The selected manoeuvre is executed by an automatic control system.

\section{Variables describing motion of the aircraft and obstacles in airspace}

The aircraft and the obstacle detected by the on-board sensing sub-system create the spatial aircraft-obstacle configuration (Becker et al., 2006; Blajer and Graffstein, 2012; Graffstein, 2012b) similar to UAV-target (Koruba and Chatys, 2005) and Missile-target (Ładyżyńska-Kozdraś, 2009). Mutual relations in this configuration are described by physical quantities illustrated in Figs. 2 and 3. These quantities apply also to each moving object separately: the aircraft with geometric centre in the point $O_{S}$ and the obstacle with geometric centre in the point $O_{P}$. Kinematic variables for the considered objects are described by mathematical relationships defined in the body axes reference system fixed to the aircraft and within the reference system fixed to the Earth. Further, the position of the aircraft towards the obstacle is considered separately (Carbone et al., 2006; Smith and Harmon, 2009): in the horizontal plane, see Figs. 2 and 4 and in vertical plane, see Figs. 3 and 5.

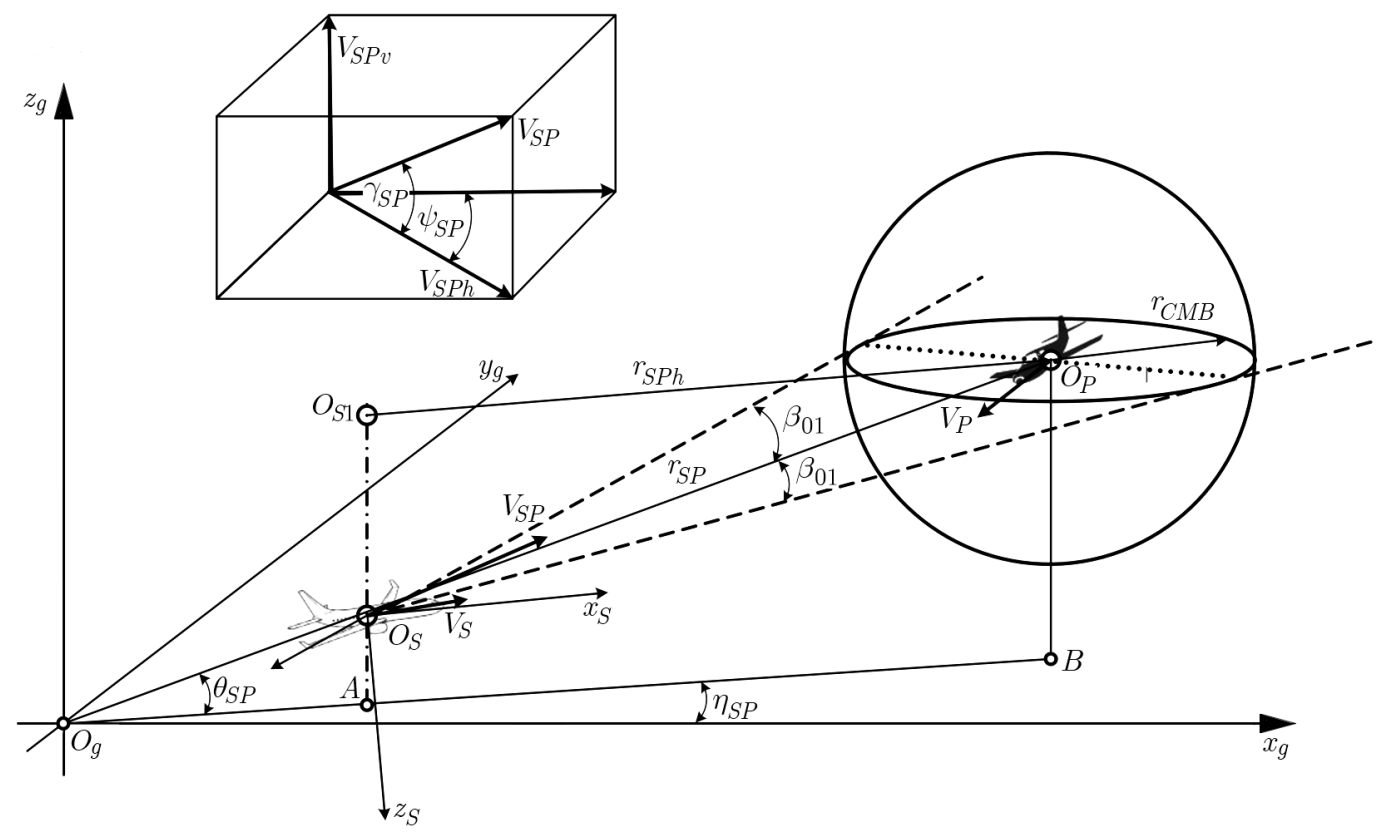

Fig. 2. The aircraft-obstacle spatial arrangement including elements contained in the horizontal plane

The linear position of the obstacle, determined in relation to the aircraft, is the important information for presented considerations. This position is described by the vector $\mathbf{r}_{S P}$ which is computed with accordance to the relationship 


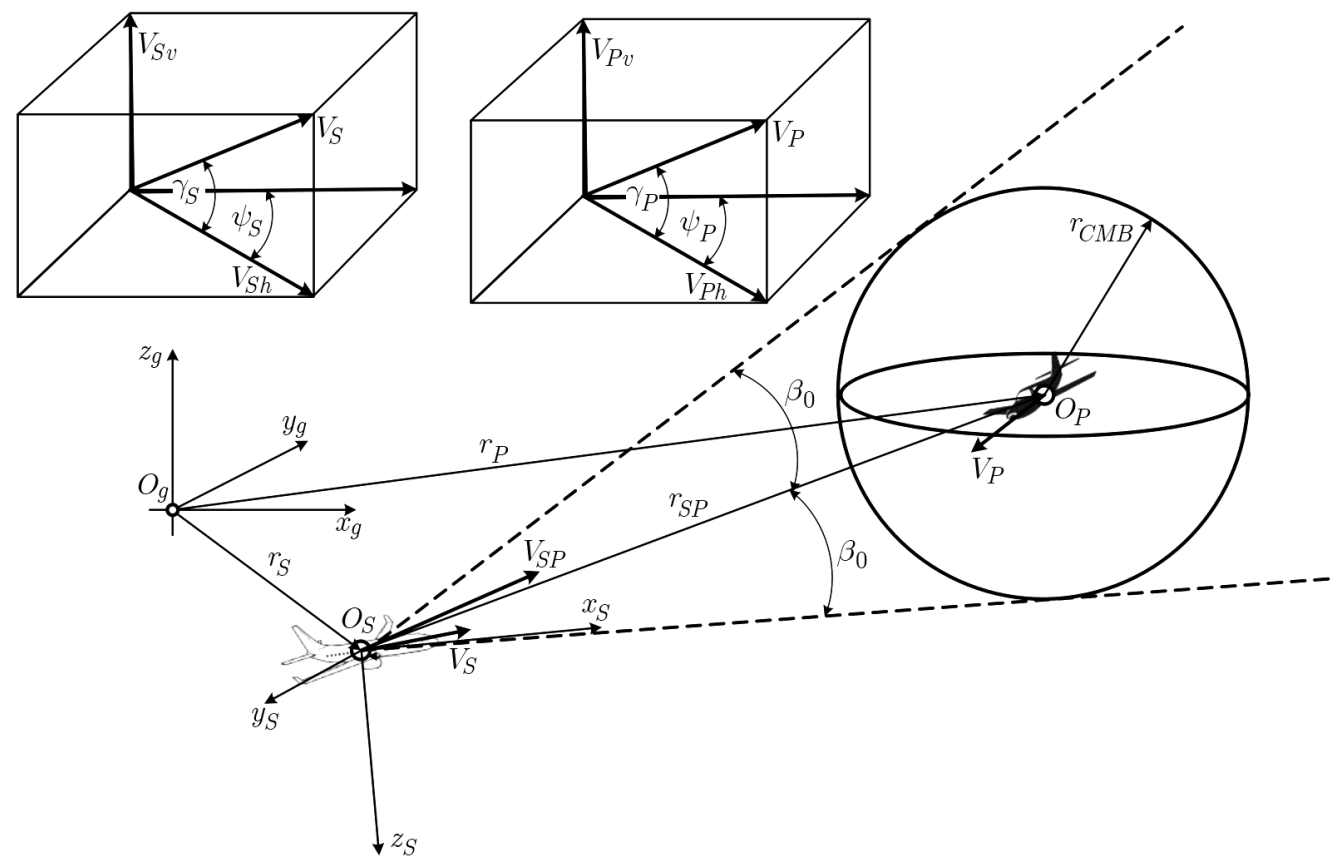

Fig. 3. The aircraft-obstacle spatial arrangement including elements contained in the vertical plane

$$
\mathbf{r}_{S P}=\mathbf{r}_{P}-\mathbf{r}_{S} \quad \mathbf{r}_{S P}=\left|\mathbf{r}_{S P}\right| \boldsymbol{\Lambda}_{r}
$$

where the matrix of transformation takes the form

$$
\boldsymbol{\Lambda}_{r}=\left[\begin{array}{lll}
\cos \gamma_{S P} \cos \psi_{S P} & \cos \gamma_{S P} \sin \psi_{S P} & -\sin \gamma_{S P}
\end{array}\right]^{\mathrm{T}}
$$

The position of the aircraft and the obstacle in the Earth reference system are

$$
\mathbf{r}_{S}=\left[\begin{array}{lll}
x_{1 S} & y_{1 S} & z_{1 S}
\end{array}\right]^{\mathrm{T}} \quad \mathbf{r}_{P}=\left[\begin{array}{lll}
x_{1 P} & y_{1 P} & z_{1 P}
\end{array}\right]^{\mathrm{T}}
$$

The aircraft-to-obstacle distance is computed according to the relationship

$$
\left|\mathbf{r}_{S P}\right|=\sqrt{\left(x_{1 P}-x_{1 S}\right)^{2}+\left(y_{1 P}-y_{1 S}\right)^{2}+\left(z_{1 P}-z_{1 S}\right)^{2}}=\frac{r_{C M B}}{\sin \beta_{0}}
$$

The components of linear velocity of the aircraft and velocity of the obstacle in the Earth reference systems and in body axes are

$$
\mathbf{V}_{i}=\left[\begin{array}{lll}
\dot{x}_{1 i} & \dot{y}_{1 i} & \dot{z}_{1 i}
\end{array}\right]^{\mathrm{T}}=\boldsymbol{\Lambda}_{V}^{-1}\left[\begin{array}{lll}
U_{i} & V_{i} & W_{i}
\end{array}\right]^{\mathrm{T}}
$$

where $i=S$ for the aircraft or $i=P$ for the obstacle.

The matrix of transformation for the aircraft and the obstacle is

$$
\boldsymbol{\Lambda}_{V i}=\left[\begin{array}{ccc}
\cos \Psi_{i} \cos \Theta_{i} & \sin \Psi_{i} \cos \Theta_{i} & -\sin \Theta_{i} \\
\sin \Phi_{i} \cos \Psi_{i} \sin \Theta_{i}-\cos \Phi_{i} \sin \Psi_{i} & \sin \Phi_{i} \sin \Psi_{i} \sin \Theta_{i}+\cos \Phi_{i} \cos \Psi_{i} & \sin \Phi_{i} \cos \Theta_{i} \\
\cos \Phi_{i} \cos \Psi_{i} \sin \Theta_{i}+\sin \Phi_{i} \sin \Psi_{i} & \cos \Phi_{i} \sin \Psi_{i} \sin \Theta_{i}-\sin \Phi_{i} \cos \Psi_{i} & \cos \Phi_{i} \cos \Theta_{i}
\end{array}\right]
$$

The trajectory angles for the aircraft and the obstacle are

$$
\gamma_{i}=\arcsin \frac{\dot{z}_{1 i}}{V_{i}}
$$


where

$$
V_{i}=\sqrt{\dot{x}_{1 i}^{2}+\dot{y}_{1 i}^{2}+\dot{z}_{1 i}^{2}}
$$

The vector of relative velocity of the obstacle is

$$
\mathbf{V}_{S P}=\mathbf{V}_{S}-\mathbf{V}_{P} \quad \mathbf{V}_{S P}=\dot{\mathbf{r}}_{S P}
$$

The components of relative velocity are (Choi and Kim, 2013)

$$
\mathbf{V}_{S P}=\left[\begin{array}{c}
V_{S P x} \\
V_{S P y} \\
V_{S P z}
\end{array}\right]=\left[\begin{array}{ccc}
\cos \gamma_{S P} \cos \psi_{S P} & -r_{S P} \cos \gamma_{S P} \sin \psi_{S P} & -r_{S P} \sin \gamma_{S P} \cos \psi_{S P} \\
\cos \gamma_{S P} \sin \psi_{S P} & -r_{S P} \cos \gamma_{S P} \cos \psi_{S P} & -r_{S P} \sin \gamma_{S P} \sin \psi_{S P} \\
-\sin \gamma_{S P} & 0 & -r_{S P} \cos \gamma_{S P}
\end{array}\right]\left[\begin{array}{c}
\dot{r}_{S P} \\
\dot{\gamma}_{S P} \\
\dot{\psi}_{S P}
\end{array}\right]
$$

The components of angular velocity of the aircraft and angular velocity of the obstacle in the body axes and Earth reference systems are

$$
\boldsymbol{\Omega}_{i}=\left[\begin{array}{lll}
P_{i} & Q_{i} & R_{i}
\end{array}\right]^{\mathrm{T}}=\boldsymbol{\Lambda}_{\Omega i}\left[\begin{array}{lll}
\dot{\Phi}_{i} & \dot{\Theta}_{i} & \dot{\Psi}_{i}
\end{array}\right]^{\mathrm{T}}
$$

where the matrix of transformation is

$$
\boldsymbol{\Lambda}_{\Omega i}=\left[\begin{array}{ccc}
1 & 0 & -\sin \Theta_{i} \\
0 & \cos \Phi_{i} & \sin \Phi_{i} \cos \Theta_{i} \\
0 & -\sin \Phi_{i} & \cos \Phi_{i} \cos \Theta_{i}
\end{array}\right]
$$

\section{Motion of the aircraft and the obstacle in the horizontal plane}

The selected horizontal plane (parallel to the surface of the Earth) includes the geometrical centre of the aircraft shifted from the point $O_{S}$ to the point $O_{S 1}$ along the straight line $A O_{S 1}$. The distance $r_{S P h}$ (in Fig. 2) to the obstacle is smaller in comparison with the real value. Physical quantities which appear to be important in the horizontal plane are illustrated in Fig. 4. The knowledge of values presented in this figure makes verification whether the threat of

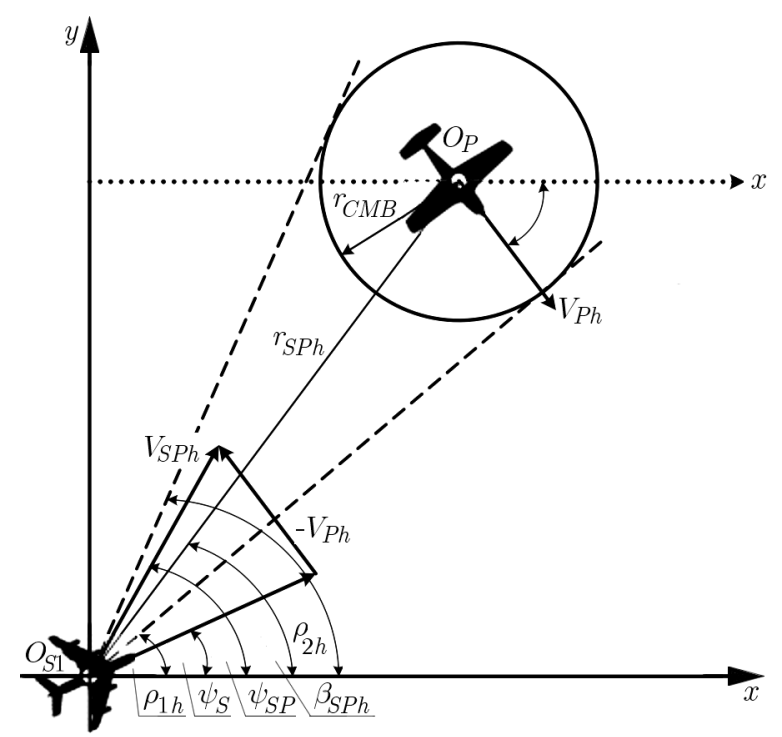

Fig. 4. The aircraft obstacle arrangement in horizontal plane 
collision with the obstacle exists in the considered plane possible. This information is the basis of the procedure of searching for the anti-collision manoeuvre (series of appropriate turns). Using trigonometric identities, the angle of the aircraft velocity vector in the horizontal plane can be described by the relationship (Paielli, 2003)

$$
\psi_{S}=\psi_{S P}+\arcsin \left[\frac{V_{P h}}{V_{S h}} \sin \left(\psi_{P}-\psi_{S P}\right)\right]
$$

The angle of relative velocity vector takes the form

$$
\psi_{S P}=\arctan \frac{V_{S h} \sin \psi_{S}-V_{P h} \sin \psi_{P}}{V_{S h} \cos \psi_{S}-V_{P h} \cos \psi_{P}}
$$

The relative velocity in the horizontal plane is equal to

$$
V_{S P h}=\sqrt{V_{S h}^{2}+V_{P h}^{2}-V_{S h} V_{P h} \cos \left(\psi_{S}-\psi_{P}\right)}
$$

where the velocity of the aircraft and obstacle in the horizontal plane is

$$
V_{i h}=\sqrt{\dot{x}_{1 i}^{2}+\dot{y}_{1 i}^{2}}
$$

The time derivative of the angle of tangent

$$
\dot{\rho}_{2 h}=\dot{\beta}_{S P h}+\dot{\beta}_{0 h}
$$

Taking into account relationships (3.4) and (4.5), the derivative of the angle of tangent is determined

$$
\dot{\rho}_{2 h}=-\frac{V_{S P h} \sin \psi_{S P}}{r_{S P h} \cos \beta_{S P h}}-\frac{\dot{r}_{S P h}}{r_{S P h}}\left(\tan \beta_{S P h}+\tan \beta_{0 h}\right)
$$

where

$$
\dot{r}_{S P h}=-\frac{r_{C M B}}{\sin \beta_{0 h} \tan \beta_{0 h}} \dot{\beta}_{0 h}
$$

and when $\theta_{S P}=\gamma_{S P}$ the equality holds $r_{S P h}=r_{S P} \cos \gamma_{S P}$.

The angle of line of sight in the horizontal plane is

$$
\beta_{S P h}=\arctan \frac{y_{1 P}-y_{1 S}}{x_{1 P}-x_{1 S}}
$$

The angles of straight lines tangent to the circle of diameter $r_{C M B}$ and centre in the point $O_{P}$ and coming through the point $O_{S I}$ included in the horizontal plane are (Benayas et al., 2002)

$$
\rho_{1 h}, \rho_{2 h}=\beta_{S P h} \mp \arcsin \frac{r_{C M B}}{\sqrt{\left(x_{1 P}-x_{1 S}\right)^{2}+\left(y_{1 P}-y_{1 S}\right)^{2}}}
$$

The aircraft-to-obstacle distance in the horizontal plane is

$$
r_{S P h}=\sqrt{\left(x_{1 P}-x_{1 S}\right)^{2}+\left(y_{1 P}-y_{1 S}\right)^{2}}=\frac{r_{C M B}}{\sin \beta_{0 h}}
$$

Verification of the reliable condition enabling determination whether collision threat exists appears to be a significant element of safe flight. The case when first two (4.10) or the last one (4.10) of inequalities presented below are fulfilled proves that the threat occurs, so the procedure according to the diagram presented in Fig. 1 ought to be initiated

$$
\psi_{S P}>\rho_{1 h} \quad \wedge \quad \psi_{S P}<\rho_{2 h} \quad \vee \quad r_{S P h}>r_{C M B}
$$


To avoid the collision in the horizontal plane, it is necessary to perform a turn of radius $r_{z s}$. During this turning, conditions regarding the derivative of the angle of relative velocity vector have to be fulfilled simultaneously. The first one of them follows from the change of the tangent angle

$$
\dot{\psi}_{S P}>\dot{\rho}_{2 h}
$$

The second condition follows from the necessity to reach the desired value of the angle of relative velocity vector before the aircraft enters the dangerous area in which the threat of collision exists

$$
\dot{\psi}_{S P}>\frac{1}{t_{Z}}\left(\psi_{S P}-\rho_{2 h}\right)
$$

The desired value of yaw angle is

$$
\Psi_{S Z}=\rho_{2 h}+\arcsin \left[\frac{V_{P}}{V_{S}} \sin \left(\psi_{P}-\rho_{2 h}\right)\right]
$$

The desired value of roll angle (Schøler et al., 2009) is

$$
\Phi_{Z}=\arctan \frac{V_{S}^{2} \cos \gamma_{S}}{g r_{z s}}
$$

\section{Motion of the aircraft and the obstacle in the vertical plane}

The spatial configuration of the vertical plane is presented in Fig. 3. Physical values, found to be necessary for considerations within this plane (Thipphavong, 2009), are shown in Fig. 5. The presented relationships have form analogous to those discussed in the previous Section, but due to different kind of aircraft motion, serious discrepancies occur in some mathematical formulas. The definition of kinematic variables enables formulation of the second condition for the collision threat. This makes it possible to prepare an anti-collision manoeuvre resulting in a change in the altitude of flight.

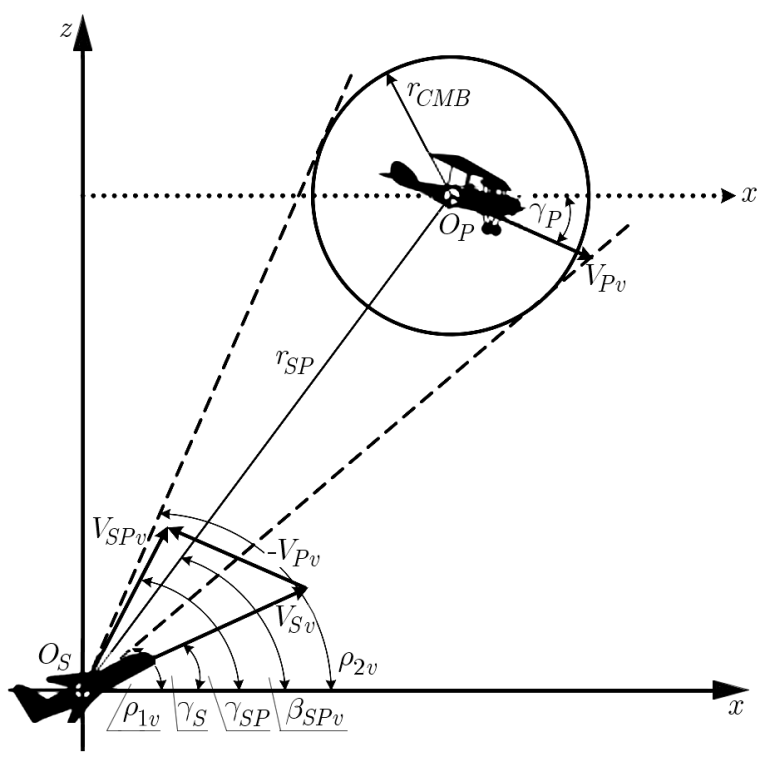

Fig. 5. The aircraft obstacle arrangement in vertical plane 
The angle of the vector of aircraft linear velocity in the vertical plane is described by a relationship analogous to (4.1)

$$
\gamma_{S}=\gamma_{S P}+\arcsin \left[\frac{V_{P v}}{V_{S v}} \sin \left(\gamma_{P}-\gamma_{S P}\right)\right]
$$

The angle of linear relative velocity vector takes the form

$$
\gamma_{S P}=\arctan \frac{V_{S v} \sin \gamma_{S}-V_{P v} \sin \gamma_{P}}{V_{S v} \cos \gamma_{S}-V_{P v} \cos \gamma_{P}}
$$

The relative velocity in the vertical plane is

$$
V_{S P v}=\sqrt{V_{S v}^{2}+V_{P v}^{2}-V_{S v} V_{P v} \cos \left(\gamma_{S}-\gamma_{P}\right)}
$$

where the velocity of the aircraft and the obstacle in the vertical plane is

$$
V_{i v}=\sqrt{\dot{x}_{1 i}^{2}+\dot{z}_{1 i}^{2}}
$$

The time-derivative of the angle of tangential line is

$$
\dot{\rho}_{2 v}=\dot{\beta}_{S P v}+\dot{\beta}_{0}
$$

Taking into account relationships (3.4) and (5.5), the derivative of the angle of the tangent line is defined by the relationship

$$
\dot{\rho}_{2 v}=-\frac{V_{S P v} \sin \gamma_{S P}}{r_{S P} \cos \beta_{S P v}}-\frac{\dot{r}_{S P}}{r_{S P}}\left(\tan \beta_{S P v}+\tan \beta_{0}\right)
$$

where

$$
\dot{r}_{S P}=-\frac{r_{C M B}}{\sin \beta_{0} \tan \beta_{0}} \dot{\beta}_{0}
$$

The angle of the line of sight in the vertical plane is

$$
\beta_{S P v}=\arctan \frac{z_{1 P}-z_{1 S}}{x_{1 P}-x_{1 S}}
$$

The angles of lines tangential to the circle of diameter $r_{C M B}$ and centre $O_{P}$, which are going through the point $O_{S}$ within the vertical plane (Benayas et al., 2002) are

$$
\rho_{1 v}, \rho_{2 v}=\beta_{S P v} \mp \arcsin \frac{r_{C M B}}{\sqrt{\left(x_{1 P}-x_{1 S}\right)^{2}+\left(z_{1 P}-z_{1 S}\right)^{2}}}
$$

Just like in the previous discussion, it is important to verify the condition of collision threat in the vertical plane. The fulfilment of first two (5.10) or the last one (5.10) of inequalities, points out that the collision threat exists, and the procedure illustrated in diagram (Fig. 1) ought to be started

$$
\gamma_{S P}>\rho_{1 v} \quad \wedge \quad \gamma_{S P}<\rho_{2 v} \quad \vee \quad r_{S P v}>r_{C M B}
$$

It is important to notice that the fulfilment of only one of logical conditions (5.10) proves that the collision threat with the obstacle exists. In the vertical plane, it is possible to avoid the collision by the climb (or descent) manoeuvre with conditions regarding the derivative of the angle of relative velocity vector $\dot{\gamma}_{S P}$ fulfilled. The first condition follows from the change of the angle of the tangent line

$$
\dot{\gamma}_{S P}>\dot{\rho}_{2 h v}
$$


The second one follows from the necessity of reaching the desired value of the angle of relative velocity before the aircraft enters the dangerous area, where the threat of collision with the obstacle is considerable

$$
\dot{\gamma}_{S P}>\frac{1}{t_{Z}}\left(\gamma_{S P}-\rho_{2 v}\right)
$$

The desired value of the trajectory angle is

$$
\gamma_{S Z}=\rho_{2 v}+\arcsin \left[\frac{V_{P}}{V_{S}} \sin \left(\gamma_{P}-\rho_{2 v}\right)\right]
$$

The desired pitch angle is

$$
\Theta_{S Z}=\gamma_{S Z}+\alpha_{S}
$$

where the angle of attack is computed by the relationship

$$
\alpha_{S}=\arctan \frac{W_{S}}{U_{S}}
$$

\section{Results of computer simulation}

The mathematical model of the I23 Manager aircraft dynamics has been used in simulations according to (Maryniak, 1992; Phillips, 2010). This model meets general, typical simplifying assumptions that were mentioned in (Maryniak, 1987).

The system of differential equations describing the aircraft motion is solved numerically within the Matlab package by the $r k 4$ procedure with a $0.01 \mathrm{~s}$ time-step.

The simulated motion of the aircraft contains two manoeuvres performed to avoid moving obstacles: climbing (within the vertical plane) and a series of turns (within the horizontal plane). A number of variables describing this motion are obtained. Appropriate time histories illustrating some selected variables describing the aircraft position, motion and control signals are also presented graphically.

Pre-determined scenarios describing motion of the objects taking part in the numerical experiment have been assumed. The obstacles are moving at constant altitudes: the first one at $160 \mathrm{~m}$, and the second one at $220 \mathrm{~m}$, respectively. Both of them perform steady, constant level motion with constant speeds with respect to the ground: $40 \mathrm{~m} / \mathrm{s}$ for the first one, $60 \mathrm{~m} / \mathrm{s}$ for the second one. The first anti-collision manoeuvre consists in climbing from the altitude of $200 \mathrm{~m}$ up to the altitude of $250 \mathrm{~m}$. The second one is composed of three turns, each one performed with the same $60^{\circ}$ roll angle. In both cases, the speed of the aircraft with respect to the ground is $50 \mathrm{~m} / \mathrm{s}$.

The aircraft and obstacle trajectories are chosen to make the considered objects come closer one to another: for the first obstacle - along perpendicular trajectories, for the second one along trajectories crossing at the angle of $40^{\circ}$. The initial positions and speeds of these objects at the moment when the evasive manoeuvring starts, guaranteed that the aircraft could perform this manoeuvres safely.

To avoid collision with an obstacle for the assumed conditions, it is required either to climb with a considerable value of the trajectory angle or to perform an alternative manoeuvre - a series of turns of appropriately small radius. In order to minimise the time needed to fly by the obstacle and return to the previous leg of trajectory, the aircraft complets the series of turns with the same pre-determined roll angle.

The flight path in the airspace is presented in Fig. 6 with symbolic representations of obstacle positions and projection of the flight path on the horizontal plane. The trajectory of the climb 


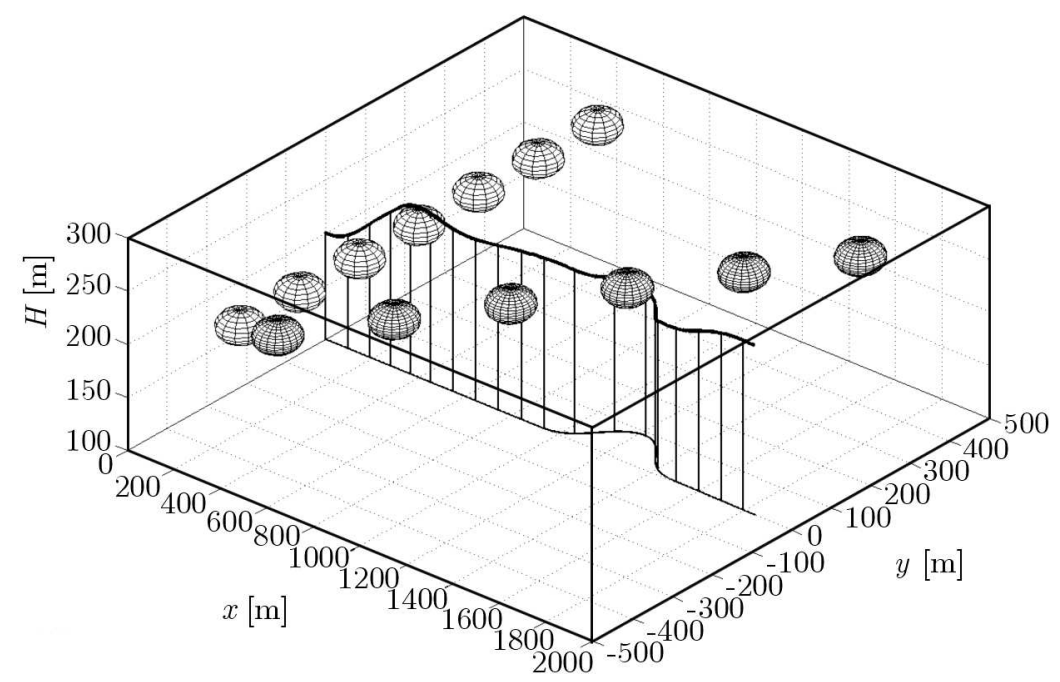

Fig. 6. Trajectories of the aircraft and obstacles in airspace

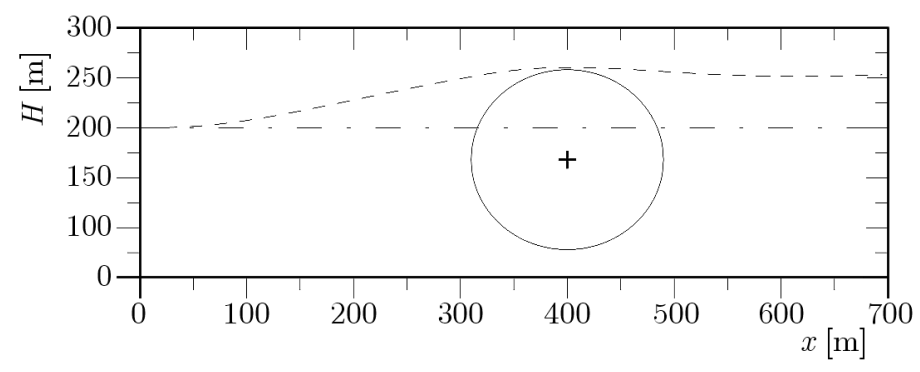

Fig. 7. The segment aircraft trajectory in the vertical plane

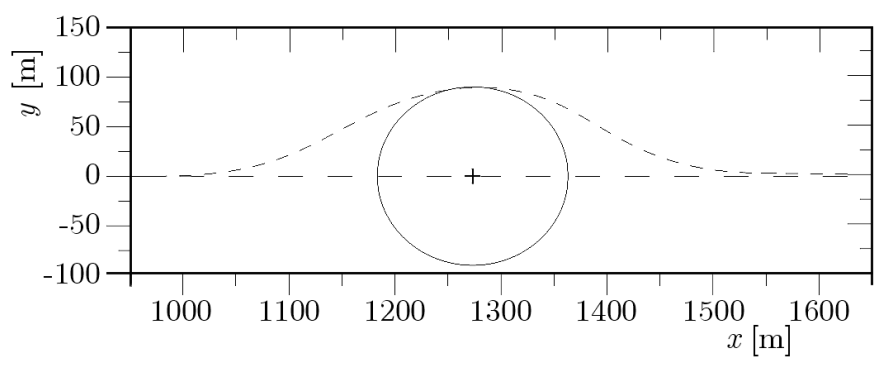

Fig. 8. The segment aircraft trajectory in the horizontal plane

manoeuvre is shown in the vertical plane in Fig. 7. The circle of $90 \mathrm{~m}$ radius represents the sum of dimensions of the aircraft, obstacle and pre-defined safety margin.

The minimum distance between the aircraft and contour of the obstacle in the vertical plane is $60 \mathrm{~m}$ what corresponds to the pre-defined safety margin. The diagram presented in Fig. 9 illustrates the time histories of pitch angle and pitch rate obtained during simulated climb. Nature of these changes follows from the elevator deflection, which is illustrated in Fig. 9. Extreme values of the elevator deflection are not exceeded.

Three turns (the first one and third to the right and the second one to the left) are necessary to omit the obstacle in the horizontal plane and then to return to the previously realised flight path (Figs. 6 and 8).

The first turn ensures avoidance of collision, the second turn - safe bypassing the obstacle, the third one - returning to the leg of the flight path realised before the anti-collision manoeuvre. The flight trajectory in the horizontal plane during the complex manoeuvre is presented in space in Fig. 6 and in the plane in Fig. 8. The safety margin has the same value: $60 \mathrm{~m}$ as in the previous 

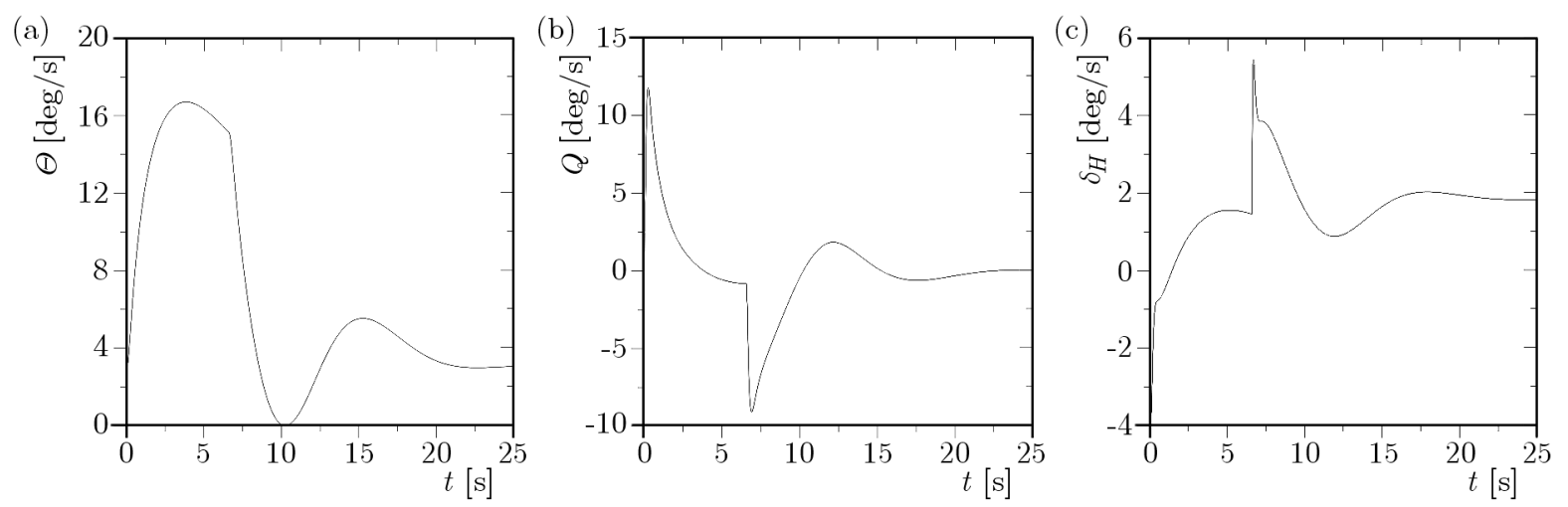

Fig. 9. The time history of pitch angle, pitch rate and the elevator deflection during the manoeuvre in vertical plane

example. The described motion of the aircraft is characterised by presented variations of the angle of the velocity vector and roll rate (Fig. 10). Aileron movements, necessary to complete the discussed complex manoeuvre, are shown in Fig. 11. Extreme values of aileron deflections vary within the range of $+15^{\circ}$ and $-15^{\circ}$. For ailerons, it is the full range of displacement. The maximum rate of the change is equal to $50^{\text {circ }} / \mathrm{s}$.
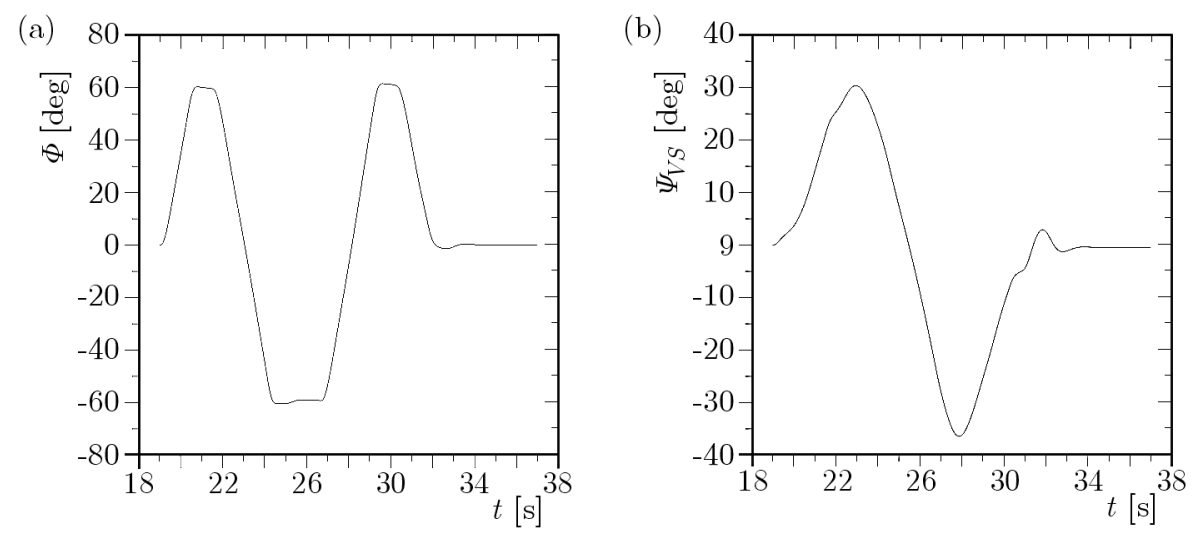

Fig. 10. The time history of roll angle and angle of velocity vector during the manoeuvre in the horizontal plane
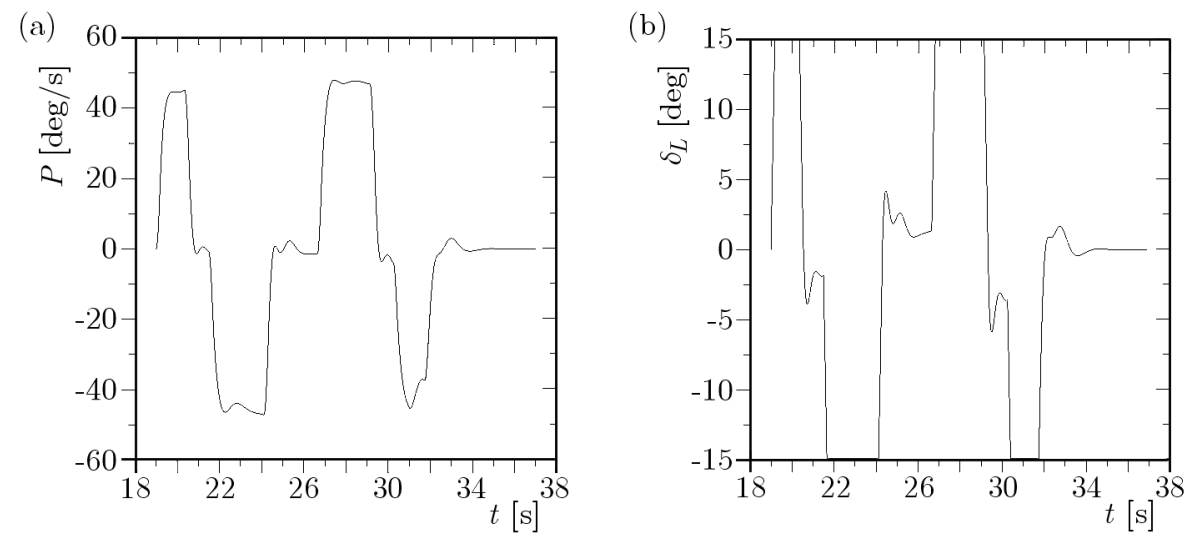

Fig. 11. The time history roll rate and aileron deflections during the manoeuvre in the horizontal plane 


\section{Concluding remarks}

In the paper, two selected scenarios of the threat of collision with a moving obstacle as well as appropriate different manoeuvres as a reaction to this threat have been presented. Discussion has been carried out on the base of the spatial scenario of the flight of the aircraft and motion of the obstacle.

During the simulated flight, two different anti-collision manoeuvres have been performed to bypass moving obstacles. Transients of state variables of the aircraft performing the anti-collision manoeuvre serve as the basis for the assessment of the object behaviour during the examined phases of flight.

Results obtained from these simulations have led to the following conclusions:

- The simulated anti-collision manoeuvres guide the aircraft to the safe state of motion keeping the accepted safety margin.

- The manoeuvre proposed in the vertical plane avoids the collision threat within $8.8 \mathrm{~s}$, whereas the manoeuvre in the horizontal plane - within $6.9 \mathrm{~s}$.

- It should be assumed that the anti-collision manoeuvre performed in the vertical plane has to be started earlier than the manoeuvre performed in the horizontal plane.

- Solution of the collision avoidance problem in the case of moving, manoeuvring obstacle may require introducing of some modifications to the presented method.

Various scenarios of motion of the aircraft and obstacle as well as their relative positions will be analysed in the future. For the assessment of the impact of disturbances typical for wind on the anti-collision manoeuvre, more results of numerical simulations are needed.

\section{References}

1. Ariyur K.B., Lommel P., Enns D.F., 2005, Reactive in flight obstacle avoidance via radar feedback, American Control Conference, Portland, 2978-2982

2. Becker M., Dantas C., Macedo W.P., 2006, Obstacle avoidance procedure for mobile robots, International Congress of Mechanical Engineering, Ouro Preto, 250-257

3. Benayas J.A., Fernández J.L., Sanz R., Diéguez A.R., 2002, The beam-curvature method: a new approach for improving local real time obstacle avoidance, The International Federation of Automatic Control, Barcelona

4. Blajer W., Graffstein J., 2012, Anti-collision manoeuvre in programmed motion theory context (in Polish), [In:] Mechanika w Lotnictwie, ML-XV 2012, K. Sibilski (Ed.), PTMTS, Warszawa, $597-613$

5. Carbone C., Ciniglio U., Corraro F., Luongo S., 2006, A novel 3D geometric algorithm for aircraft autonomous collision avoidance, IEEE Conference on Decision and Control, San Diego, $1580-1585$

6. Choi H., Kiм T.Y., 2013, Reactive collision avoidance of unmanned aerial vehicles using a single vision sensor, Journal of Guidance, Control, and Dynamics, 36, 4, 1234-1240

7. Fasano G., Forlenza L., Accardo D., Moccia A., 2010, Integrated obstacle detection system based on radar and optical sensors, AIAA Infotech and Aerospace Conference, Atlanta, 1-17

8. Freeman P., Moosbrugger P., 2010, A low cost phased array solution for UAV collision avoidance, AIAA Infotech and Aerospace Conference, Atlanta, 1-4

9. Graffstein J., 2006, Changes in automatically controlled object motion caused by selected perturbations (in Polish), [In:] Mechanika w Lotnictwie, ML-XII 2006, J. Maryniak (Ed.), PTMTS, Warszawa, 103-119 
10. Graffstein J., 2012a, Anti-collision manoeuvre: process and parameters selection (in Polish), Transaction of the Institute of Aviation, 224, 32-45

11. Graffistein J., 2012b, Elements of collision threat detection process and automatically controlled emergency manoeuvre (in Polish), Measurements, Automation, Robotics, 2, 383-387

12. Higuchi T., Toratani D., Ueno S., 2012, Double tetrahedron hexa-rotorcraft collision avoidance of indoor flying, International Congress of the Aeronautical Sciences ICAS, 1-7

13. Koruba Z., Chatys R., 2005, Gyroscope-based control and stabilization of unmanned aerial mini-vehicle (mini-UAV), Aviation, 9, 10-16

14. Lalish E., Morgansen K.A., Tsukamaki T., 2009, Decentralized reactive collision avoidance for multiple unicycle-type vehicles, American Control Conference, Seattle, 5055-5061

15. ŁADYŻYŃSKA-KOZDRAŚ E., 2009, The control laws having a form of kinematic relations between deviations in the automatic control of a flying object, Journal of Theoretical and Applied Mechanics, 47, 2, 363-381

16. MARYNIAK J., 1987, The system of airplane flight simulation for homing and air combat (in Polish), Journal of Theoretical and Applied Mechanics, 25, 1/2, 189-214.

17. MARYNIAK J., 1992, General mathematical model of controlled aircraft (in Polish), [In:] Mechanika w Lotnictwie, J. Maryniak (Ed.), PTMTS, Warszawa, 575-592

18. PAielli R.A., 2003, Modeling maneuver dynamics in air traffic conflict resolution, Journal of Guidance, Control, and Dynamics, 26, 3, 407-415

19. PARK J.-W., Kim J.-H., TAHK M.-J., 2012, UAV collision avoidance via optimal trajectory generation method, International Congress of the Aeronautical Sciences ICAS, Brisbane, 1-7

20. Phillips W.F., 2010, Mechanics of Flight, John Willey \& Sons, Inc.

21. Schøler F., la Cour-Harbo A., Bisgaard M., 2009, Collision Free path generation in 3D with turning and pitch radius constraints for aerial vehicles, AIAA Guidance, Navigation, and Control Conference, Chicago, 1-11

22. Seo J., Kim Y., Tsourdos A., White B.A., 2012, Multiple UAV formation reconfiguration with collision avoidance guidance via differential geometry concept, International Congress of the Aeronautical Sciences ICAS, Brisbane, 1-8

23. Smith A.L., Harmon F.G., 2009, UAS collision avoidance algorithm minimizing impact on route surveillance, AIAA Guidance, Navigation, and Control Conference, Chicago, 1-20

24. Thipphavong D., 2009, Analysis of a multi-trajectory conflict detection algorithm, for climbing flights, AIAA Aviation Technology, Integration, and Operations Conference, Hilton Head, 1-13 